IEEE/AIAA $27^{\text {th }}$ Digital Avionics Systems Conference, October'08, St. Paul, Minnesota, USA

\title{
FLEXIBLE ELECTRICAL MANAGER SERVICE FOR UAS APPLICATIONS DEVELOPMENT
}

\author{
Pablo Royo, Juan López, Enric Pastor and Cristina Barrado \\ Department of Computer Architecture, Technical University of Catalonia \\ 08860 Castelldefels (Barcelona), Spain
}

\begin{abstract}
Unmanned Aerial System (UAS) are becoming viable aerial platforms for civil oriented monitoring applications. However, in most cases the selected UAS platforms are ad-hoc vehicles which include highly heterogeneous avionics. Avionics on-board may be COSTS modules that have highly different non-standard power requirements. Additionally, the available power sources in these UAS may be fairly limited or even restricted to battery units.

This paper introduces the ELectrical Manager Service (ELMS), an on-board system in charge of offering a flexible power supply architecture that supports minimal reconfiguration overhead for a wide variety of UAS missions. The ELMS Service is designed to offer a continuous monitoring of the state of the power network, and a coherent and controlled response in front of power supply contingencies. The ELMS will monitor the batteries and generator status, the power consumption of the avionics and other systems, manage the connection/disconnections of systems, and provide power availability estimations. The ELMS Service is part of an architecture designed to facilitate the execution of UAS civil missions, the USAL. The USAL is built as a set of cooperating services in a purely distributed and scalable architecture with a middleware that manages inter-service communications.
\end{abstract}

\section{Introduction}

An Unmanned Aerial System (UAS) is an expression that identifies an aircraft that can fly without a pilot on board; that is, an airframe and a computer system which combines sensors, GPS, servos, CPUs, etc. With the avionics technological evolution, Unmanned Aerial Systems are also becoming a valid option for civil applications in addition to their traditional military role. UAS could be used to perform environmental monitoring applications, help in emergency situations, support surveillance operations, operate as communication relays and much more.

Most of these civil UAS operations are implemented by ad-hoc UAS airframes that include heterogeneous payload requirements in order to implement the target missions. Many different Consumer off-the-shelf (COTS) devices are usually included on-board, which have different power supply requirements, i.e. the autopilot, sensors, engines, cameras, CPUs, hard disks, communication modules, etc. All of them may eventually take part in an UAS mission.

Additionally, many UAS are limited to operate on battery power or have a limited power generation capacity. Hence, in any phase of the UAS mission, several payload modules or even all of them might be operating at the same time. This may be a problem if they need to work for a long time or if their combined power requirement exceeds the available power capacity. Therefore the power management during the UAS mission may become a critical part of the system.

In order to build economically and mission viable UAS solutions, the same power supply platform should be able to implement a variety of organizations with minimal reconfiguration overhead. Also, not only a well designed power supply network is required, but a high level of monitoring and control capacity is necessary to allow a fine tuning of the system execution to keep the overall power budget within its nominal parameters.

In case of electrical contingencies and to safely end the mission, some payload modules may have to be switched off just to save energy in order to keep working, for example, the autopilot or some communication module. Therefore, to guarantee that critical payload devices keep operating or the security of the flight or the mission, electrical contingency management must be available. 
IEEE/AIAA $27^{\text {th }}$ Digital Avionics Systems Conference, October'08, St. Paul, Minnesota, USA

This paper presents a new UAS subsystem, called the ELectrical Manager Service (ELMS), specially designed to monitor and control the power supply in an UAS. The ELMS Service is designed to offer a continuous monitoring of the state of the power network and a coherent and controlled response in front of power supply contingencies as we have already mentioned.

The ELMS Service is part of an architecture designed to facilitate the execution of UAS civil missions, the USAL [1]. The USAL is built as a set of cooperating services on-board the UAV and on the ground, connected by a Local Area Network (LAN), in a purely distributed and scalable architecture. These services cooperate for the accomplishment of the UAS mission. They rely on a middleware that manages and communicates the services [2].

The ELMS is part of the Flight Service Category in the USAL and will monitor and control the power supply network on-board the UAS on behalf the Contingency Management Service. More precisely, the ELMS is in charge of:

- Monitor the batteries status.

- Monitor and start and stop the alternators of the system.

- Monitor the behavior of the power consumers.

- Switch off/on the power consumers.

- Monitor and manage the power connections.

The rest of the paper is organized as follows: Section2 introduces the underlying UAV Service Abstraction Layer (USAL) in which the ELMS operate. Section 3 overviews the global objectives of the ELMS, the power distribution network assumed on-board the UAV and the main design elements. Section 4 introduces all elements necessary for a proper power source monitoring, including batteries and power generators. The characterization of power consumers is described in Section 5. Power producers and consumers can be connected through a dynamic power distribution network described in Section 6. The high-levels functionalities offered by the ELMS together with its relation with the flight dispatching process are outlined in Section 7. Finally, Section 8 concludes the paper and outlines future research.

\section{UAV Service Abstraction Layer Overview (USAL)}

For executing UAS civil missions, we proposed a distributed embedded system that will operate both on-board the airframe and at the ground station and that will operate as a payload/mission controller. Over the different distributed elements of the system, we deploy software components, called services, which implement the required functionalities. These services cooperate for the accomplishment of the UAS mission. They rely on a middleware that manages and communicates the services. The communication primitives provided by the middleware promote a publish/subscribe model for sending and receiving data, announcing events and executing commands among services [2].

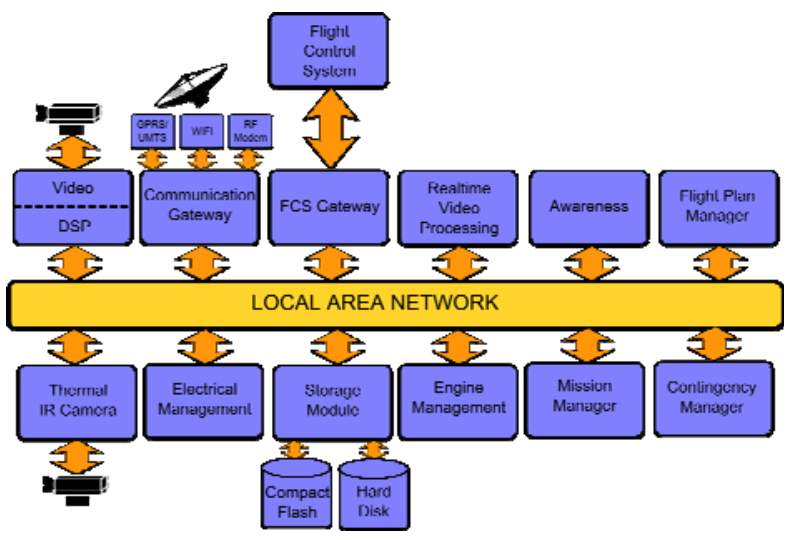

Figure 1. Overview of the USAL Service-based Architecture

As it can be seen in Figure 1, the different components of the UAS are interconnected by a local network that makes data communication between them very flexible and efficient.

In order to keep low the development and maintenance costs for UAV systems, a common infrastructure is provided for communicating UAS services. The existence of an open-architecture avionics package specifically designed for UAS may alleviate the costs by reducing the development to a simple parameterization.

The design of this open-architecture avionics system starts with the definition of its requirements. From the study and definition of several UAS missions, one can identify the most common requirements that are present among them. These 
IEEE/AIAA $27^{\text {th }}$ Digital Avionics Systems Conference, October'08, St. Paul, Minnesota, USA

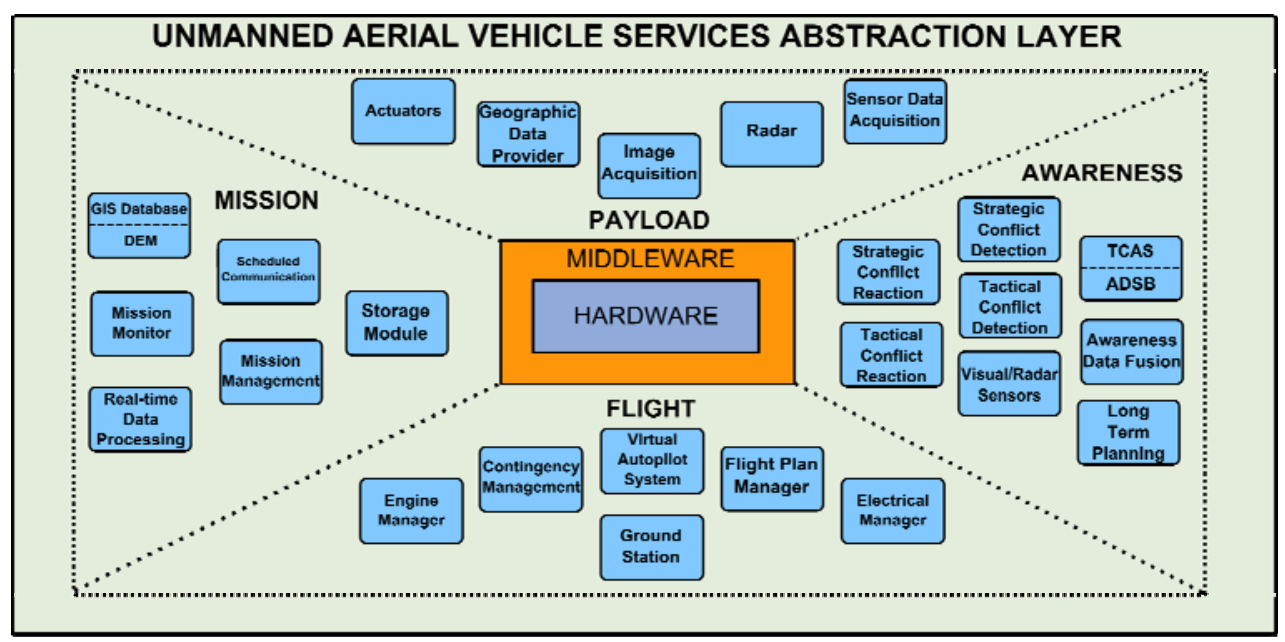

Figure 2. Overview of the USAL service-based architecture.

common functionalities have to be organized and implemented in an abstraction layer called UAS Service Abstraction Layer (USAL) [1].

The UAS Service Abstraction Layer (USAL) is the set of available services running on top of the UAV System architecture to give support to most types of UAS civil missions. USAL can be compared to an operating system. Computers have hardware devices used for input/output operations. Every device has its own particularities and the OS offers an abstraction layer to access such devices in a uniform way. Basically, it publishes an Application Program Interface (API) which provides end-users with efficient and secure access to all hardware elements. The USAL is a software abstraction layer that gives facilities to end-users programs to access the UAS payload.

The final goal of the USAL is twofold:

- First, reduce "time to market" when creating a new UAS system.

- Second, simplifying the development of all systems required to develop the actual mission assigned to the UAS.

USAL has its services divided in several categories. Services are presented in a same category when they cooperate in the same main objective, such as fight, mission, payload or awareness. Available services have been classified in four categories.
Flight services: all services in charge of basic UAS fight operations: autopilot, basic monitoring, contingency management, etc.

Mission services: all services in charge of developing the actual UAS mission.

Payload services: specialized services interfacing with the input/output capabilities provided by the actual payload onboard the UAS.

Awareness services: all services in charge of the safe operation of the UAS related to terrain avoidance and integration with shared airspace.

Figure 2 provides a global vision of most services that can take part in a UAS mission, most of them already predefined in the USAL.

\section{Flight Service Category}

The flight category is in charge of basic UAS fight actions. This category is composed of several services where each of them has a specific goal. Figure 3 shows the most important flight services and how they are interconnected.

The Virtual Autopilot Service (VAS) is a service that interacts with the selected autopilot and therefore it needs to be adapted to its peculiarities. The VAS will operate similarly as drivers work on operating systems, abstracting away the implementation details from actual autopilot users.

The Flight Plan Manager Service is designed to implement much richer flight-plan capabilities 
IEEE/AIAA $27^{\text {th }}$ Digital Avionics Systems Conference, October'08, St. Paul, Minnesota, USA

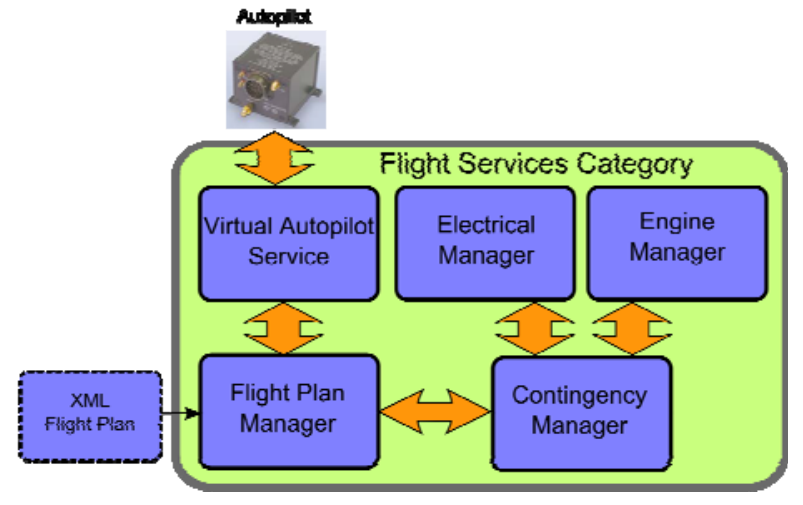

Figure 3. Overview of the available services in the flight service category.

that those available capabilities offered by the current autopilot [3].

The Contingency Manager Service is responsible of collecting status information related to multiple sources: engine, electrical, fuel, communications, mission, etc, identifying if some type of contingency has evolved and deciding which type of reaction is required.

The Engine Manager Service is in charge of monitoring the engine parameters of the UAS in order to detect problems and notify the corresponding contingencies.

Finally, the Electrical Manager Service (ELMS) is in charge of monitoring the electrical parameters of the UAS. The ELMS is specially designed to control the power supply in an UAS. Next sections detail the ELMS objectives and design deeply.

\section{ELMS Objectives and Design}

The ELMS is an important component inside the USAL flight category. This service is in charge of managing all the electrical capacities in the UAS. With ELMS several design objectives are pursued.

\section{ELMS Objectives}

The ELMS should provide a flexible platform to solve the wide diversity power supply requirements in a UAS. Devices as cameras, sensors, RF modules, CPUs, etc may have different power supply requirements. Additionally, the available power supply elements in the UAS may be quite diverse, different types of batteries with specific capacities and voltage ranges, alternators, solar panels, etc. Therefore, the ELMS operate with an underlying organization (see Figure 4). Electrical power can be provided either by batteries and/or diverse power generators. Power can be directly operated by consumers or transformed through voltage regulators. Finally, all these elements are interconnected via a number of configurable power distribution networks. Each one of these elements is managed by the ELMS Service.

In order to manage the power supply system it is basic to know the status of all modules providing energy to the power network. The ELMS needs to monitor the different batteries in the UAS and know what their features are. Another requirement of the ELMS is to keep operating the different payload modules during the mission. That means that in certain cases it may be necessary to switch a low battery in a low status for an alternative one, or to charge a battery using a power source. Also it is necessary to monitor the inter-connections status to change the connection configuration if necessary.

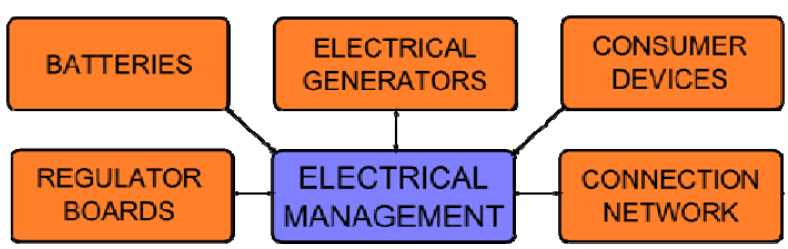

Figure 4. Organization of the power network onboard the UAS.

While monitoring the overall electrical parameters of the UAS, the ELMS can detect problems and notify any power contingency. When the whole network is operating properly the ELMS can provide several maintenance responses as for example:

- The time which we can remain with the current mission.

- If critical devices as, for example, the autopilot, runs any sort of power risk.

- If we can incorporate any device along the mission, without content at risk the rest of the systems.

- Only save energy, switch on the devices when it is necessary. 
IEEE/AIAA $27^{\text {th }}$ Digital Avionics Systems Conference, October'08, St. Paul, Minnesota, USA

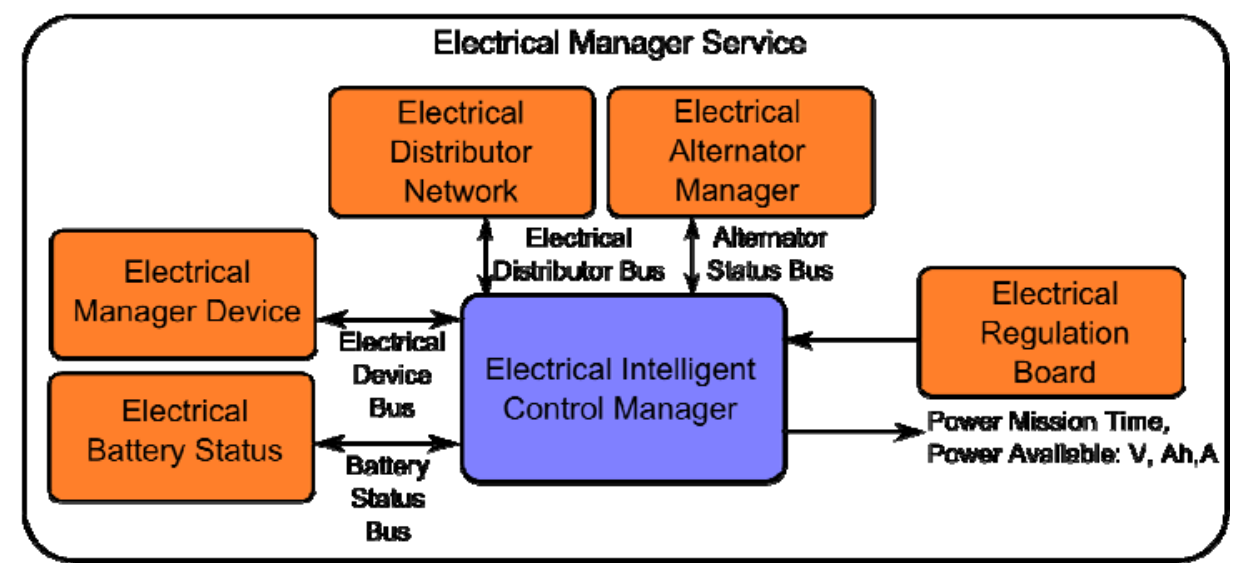

Figure 5. Electrical Manager Service Interaction.

\section{ELMS Design Guidelines}

The ELMS is a distributed service. This means that, the service has external subservices which provide the power parameters from the power consumers and providers. So, we allocate these subservices next to the batteries, the alternators and the consumers, as for example cameras, CPU, etc. Subservices will send the information to the ELMS central node in order to process it and offer intelligent responses. Sometimes we will need to configure the power supply connections, just to switch on/off any device or to select any battery. This task will be carried out with an interconnection matrix. This matrix is composed by power transistors.

One subservice alone does not have a great value; however with a perfect orchestration of them, we obtain a highly capable platform that can be easily integrated to perform very efficiently the power supply management.

Figure 5 shows the interaction between different components of the service. The figure shows that each component has its own data bus in order to interact with the electrical intelligent control manager; however this implementation can change. One data bus could be shared with more than one component. So this figure is one of the possible implementations.

\section{Power Source Monitoring}

The ELMS Service is in charge of monitoring all possible energy sources on-board the UAS.
These should include multiple batteries but also onboard generators, solar cells or any other type of power source. Currently, ELMS has models to monitor batteries and generators.

\section{Electrical Battery Status}

Identifying exactly how much energy is left in a battery is not an easy task. A voltmeter is useful but it will only indicate that the battery is flat when it may be too late. In order to keep an accurate record of what is stored in a battery it is necessary to keep account of all the factors that affect it.

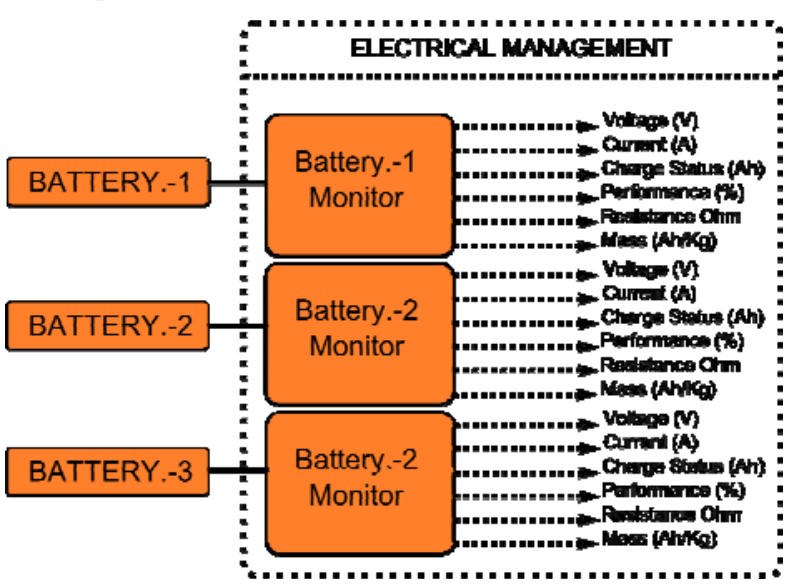

Figure 6. Battery Monitor Architecture.

To characterize a battery it is necessary to know more parameters than just voltage $[4,5]$. To achieve this goal we have to monitor parameters such as: voltage, current, battery charge status, energy stored, battery resistor, battery mass and battery efficiency. 
IEEE/AIAA $27^{\text {th }}$ Digital Avionics Systems Conference, October'08, St. Paul, Minnesota, USA

Table 1 Batteries Comparative.

\begin{tabular}{l|c|c|c|c|c|}
\hline Type & Energy/mass & V/element & $\begin{array}{c}\text { Number of } \\
\text { recharges }\end{array}$ & Time of charge & $\begin{array}{c}\text { Auto-discharge } \\
\text { per month (\% of total) }\end{array}$ \\
\hline \hline $\mathrm{Pb}$ & $30-50 \mathrm{Wh} / \mathrm{kg}$ & $2 \mathrm{~V}$ & $200-300$ & $8-16 \mathrm{~h}$ & $5 \%$ \\
\hline $\mathrm{Ni}-\mathrm{Cd}$ & $48-80 \mathrm{Wh} / \mathrm{kg}$ & $1,25 \mathrm{~V}$ & 1500 & $1 \mathrm{~h}$ & $20 \%$ \\
\hline $\mathrm{Ni}-\mathrm{H}$ & $60-120 \mathrm{Wh} / \mathrm{kg}$ & $1,25 \mathrm{~V}$ & $300-500$ & $2 \mathrm{~h}-4 \mathrm{~h}$ & $30 \%$ \\
\hline $\mathrm{Li}-\mathrm{ion}$ & $110-160 \mathrm{Wh} / \mathrm{kg}$ & $3,6 \mathrm{~V}$ & $500-1000$ & $2 \mathrm{~h}-4 \mathrm{~h}$ & $10 \%$ \\
\hline $\mathrm{Li}-\mathrm{Po}$ & $100-130 \mathrm{Wh} / \mathrm{kg}$ & $3,6 \mathrm{~V}$ & $300-500$ & $1 \mathrm{~h}-1.5 \mathrm{~h}$ & $10 \%$ \\
\hline
\end{tabular}

Some of these parameters are inherent in batteries however some of them will have to be monitored in real time to know current battery status.

As it is shown in the Figure 6, one monitor is deployed in each battery, providing all required parameters for each battery on-board the UAS. These monitors send all the status information to the intelligent control manager. The ELMS central process data publishes the batteries status to the rest of the network.

Battery status subsystems analyze the following basic parameters (see Table 1) assuming that available support exists for hardware-assisted monitors:

- Voltage (Volts).

- Current (Ampere)

- Battery Charge Status in ampere-hour (Ah).

- The Energy stored in Watt-hour.

- The Battery resistor in Ohm.

- $\quad$ Battery mass in (Ah/Kg).

- $\quad$ The battery efficiency in percentage (\%).

\section{Electrical Alternator Manager}

An alternator offers the possibility to increase the UAS power supply autonomy. In order to manage an alternator we have designed the Electrical Alternator Manager. An alternator offers two possibilities: one use as source of power supply and another use as battery charger.

The Electrical Alternator Manager is in charge of continuously monitoring the proper operation of the alternator, keeping track of the maximum amount of current it can provide and of the actual amount of current that is drawn from it. Keeping its operation within these limits, the current provided by this power source can be redirected both to charge batteries and to provide current to operative avionics (see Figure 7). Batteries and avionics can be connected to an alternator through an Electrical Distribution Network controlled by an equivalent number of digitally controlled power switches.

Based on the dynamic evolution of the UAS mission and the power requirements at each stage, the ELMS will connect/disconnect the control signals distributing the alternator current to those subsystems that require it.

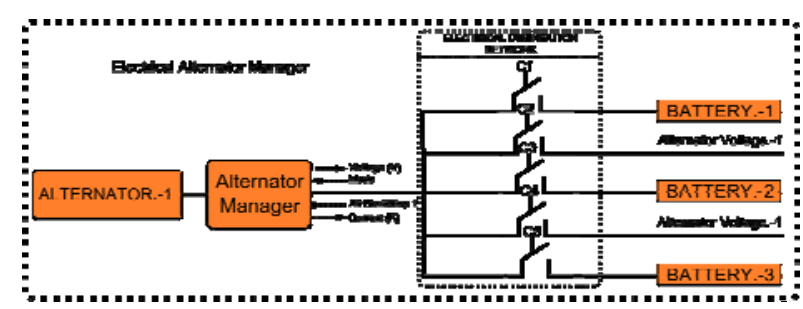

Figure 7. Architecture of the Electrical Alternator Manager.

\section{Electrical Device Manager}

The avionics and the entire payload on-board the UAS need power to properly operate. Each one of these devices has different power requirements in terms of acceptable voltage ranges and dynamic current and power consumption. Hence, it is very important to properly characterize these requirements for each consumer and the real time power status in order to offer a coherent picture of the overall power budget in the system $[7,8]$. This 
IEEE/AIAA $27^{\text {th }}$ Digital Avionics Systems Conference, October'08, St. Paul, Minnesota, USA

information will also allow precise predictions of the future evolution of this power budget and eventually an intelligent response in case of electrical contingencies. The Electrical Device Manager pursues several objectives.

First, the Electrical Device Manager should publish how much current is consuming each device at every moment. This permits to compute the available power supply left at the batteries or if the current supplied by generators is surpassed. Also, device malfunctions can be detected if any device consumes more energy than it nominally should do; thus leading to device failure.

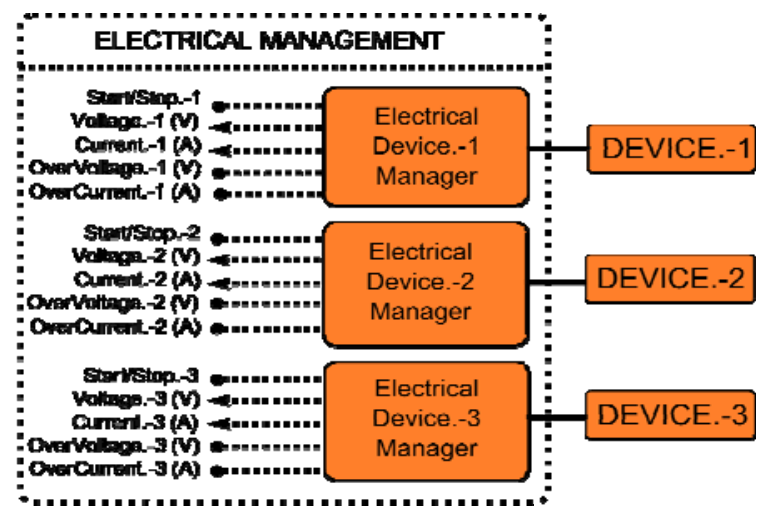

Figure 8. Electrical Device Manager Architecture.

Second, it provides the device electrical parameters, as for example: voltage thresholds and average/peak current and power consumption.

Third, it offers an electrical circuit protection in front of over-voltage and over-current. The electrical device manager informs the electrical intelligent control about the problem in order to shut down or disconnect the device.

Finally the Electrical Device Manager fourth goal is related to managing the device power supply, in order to turn it on/off on demand (according to the mission requirements). Figure 8 shows the Electrical Device Manager architecture. In this case we represent three devices.

Once the Electrical Battery Status, the Electrical Alternator Manager and the Electrical Device Manager are available to monitor the electrical sources and sinks, we can make interesting decisions to manage the available power. However, in order to offer the correct power supply to any device in the system we need an electrical network to distribute power over the UAS.

\section{Electrical Distribution Network}

The Electrical Distribution Network connects the power producers and power consumers. The goal is to develop a flexible platform which will make possible to interconnect any input with any output and to dynamically select among the available connections.

Figure 9 shows a simple example of an Electrical Distributor Network configuration. In this example we want to select one among five available batteries (inputs). In the figure; by means of a number of control signals we can configure which is the battery that drives a given output. In some cases, one battery may drive more than one output, but each output can only be driven by a single power source.

In general, not all potential interconnections will be available due to wiring limitations or due to incompatible voltage ranges. Batteries have a voltage range determined by their nominal voltage and discharge curve; while consumers have voltage ranges determined by their input regulators. Incompatible ranges should never be connected to prevent module failure.

\section{Electrical Intelligent Control Manager}

When all accessory subservices are available, monitoring batteries, alternators, devices, and connections; the Electrical Intelligent Control Manager will be able to provide several elaborated responses. For example:

- The time which we can remain with the actual mission configuration.

- Identify contingencies related to critical devices, as for example if the autopilot is in risk of losing a reliable power source due to the activation of excessive payload modules.

- $\quad$ It will permit to save on-board energy by switching on and off payload modules only when it is necessary. 
IEEE/AIAA $27^{\text {th }}$ Digital Avionics Systems Conference, October'08, St. Paul, Minnesota, USA

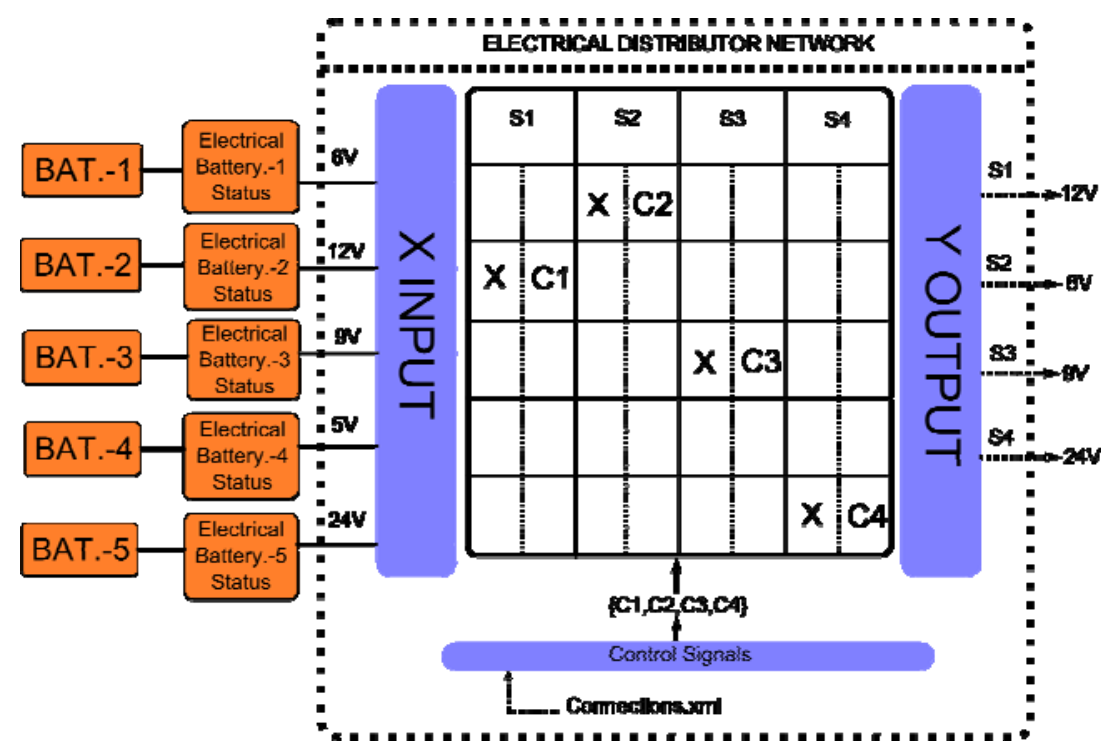

Figure 9 Electrical Distributor Network Architecture.

The high level architectural view of the electrical system, together with this elaborated information is a fundamental part of the flight dispatching of the UAS [6]. The ELMS provides a structured view of the electrical system on-board the UAS, allowing nominal mission time predictions, but also identifying all potential sources of risk in case of battery or generator failure. Under such degraded modes predictions are still available, thus permitting the contingency manager to identify the correct flight route to a safe landing in terms of available flight-time.

In case of contingencies identified by the ELMS or by any other subsystem, a coherent response can be provided by switching off those payload modules that have been identified as noncritical for the flight.

\section{Conclusions}

This paper has introduced the Electrical Manager Service (ELMS) as a service inside the underlying UAV Service Abstraction Layer (USAL), The ELMS provides a well structured view of the electrical system on-board an UAS and offers a number of functionalities to keep a continuous monitoring on the critical parameters of both power sources and power consumers. The detailed monitoring of the power budget on-board the system permits identifying energy contingencies that will be informed to the contingency manager for a proper coordinated response. Dynamic power reconfigurations can also be managed, allowing the power distribution network to be adapted to each phase of the flight or even to detected contingencies.

As future work additional models will be included for other power sources like solar cells, and the full integration with the contingency manager will be designed by pre-defining the set of global contingencies that can occur in a UAS and pre-setting the appropriate reaction to each one.

\section{References}

[1] Pastor, E. et al., June 2007, "UAV Payload and Mission Control Hardware/Software Architecture”, Aerospace and Electronic Systems Magazine, IEEE, vol.22, no.6, pp.3-8.

[2] J. Lopez, P. Royo, E. Pastor, C. Barrado, and E. Santamaria, "A middleware architecture for unmanned aircraft avionics,” ACM/IFIP/USEUNIX 8th Int. Middleware Conference, NewPort, California, Nov. 2007.

[3] E. Santamaria, P. Royo, J. Lopez, C. Barrado, E. Pastor, and X. Prats, "Increasing UAV capabilities through autopilot and flight plan abstraction," Proceedings of the 26th Digital Avionics Systems Conference, Dallas, Texas, 2007. 
IEEE/AIAA $27^{\text {th }}$ Digital Avionics Systems Conference, October'08, St. Paul, Minnesota, USA

[4] Daler N. Rakhmatov and Sarma B. K. Vrudhula, "An analytical high-level battery model for use in energy management of portable electronic systems”, IEEE/ACM international conference on Computer-aided design, San Jose, California, 2001.

[5] Hongqing Cao, Jingxian Yu, Lishan Kang, Hanxi Yang and Xinping Ai, "Modeling and prediction for discharge lifetime of battery systems using hybrid evolutionary algorithms”, Computers \& Chemistry, Vol. 25, Issue 3, pp 251-259, 2001.

[6] X. Prats, E. Pastor, P. Royo and J. Lopez, "Flight dispatching for unmanned aerial vehicles" Proceedings of the AIAA Guidance, Navigation, and Control Conference, Honolulu, Hawaii, 2008.

[7] Chun-Hao Hsu, Jian Jhen Chen,; Shiao-Li Tsao, "Evaluation and modeling of power consumption of a heterogeneous dual-core processor"; International Conference on Parallel and Distributed Systems, Vol. 1, 5-7, Dec. 2007.

[8] J. Zedlewski, et al. "Modeling Hard-Disk Power Consumption”, Proceedings of the 2nd USENIX Conference on File and Storage Technologies, pp: 217-230, 2003.

\section{Acknowledgements}

This work has been partially funded by Ministry of Science and Education of Spain under contract TIN 2007-63927.

27th Digital Avionics Systems Conference October 26-30, 2008 